\title{
The micellization and dissociation transitions of thermo-, pH- and sugar-sensitive block copolymer investigated by laser light scattering
}

\author{
Y. C. Tang*, J. H. Wu, J. X. Duan \\ College of Chemistry and Materials Science, Anhui Normal University, 241000 Wuhu, China
}

Received 5 December 2011; accepted in revised form 25 February 2012

\begin{abstract}
A triple-stimuli responsive polymer, poly(3-acrylamidophenylboronic acid)-b-poly(N-isopropylacrylamide) (PAAPBA-b-PNIPAM), has been synthesized by reversible addition-fragmentation chain transfer polymerization. Temperature, $\mathrm{pH}$, and fructose induced micellization and dissociation transition of block copolymer was investigated by a combination of static and dynamic laser light scattering. PAAPBA-b-PNIPAM copolymer self-assembles into micelles with PAAPBA block as core and PNIPAM as shell in lower $\mathrm{pH}$ aqueous solution at room temperature. Increasing the temperature causes the micelle to shrink due to the dehydration of PNIPAM segments at $\mathrm{pH}$ 6.2. After the elevation of solution $\mathrm{pH}$ from 6.2 to 10.0, the increase in the hydrophilicity of PAAPBA block leads to an expulsion of unimers from micelles. In addition, the fructose addition further enhances the dissociation of micelles. Our experiments demonstrate that the micelle to unimer transition process proceeds via the step-by-step sequential expulsion of individual chains.
\end{abstract}

Keywords: smart polymers, PAAPBA-b-PNIPAM, micelle dissociation, laser light scattering

\section{Introduction}

Stimuli-responsive block copolymers have attracted considerable interest over the past decades because of their potential applications in drug delivery, biosensors, and nanotechnology [1]. This kind of polymers can self-assemble into micelles or socalled inverse micelles with desirable morphologies and properties in response to external stimuli, such as $\mathrm{pH}$, ionic strength, and temperature. In recent papers, some examples of triple-stimuli responsive copolymers were described, which would independently modulate their response to each stimulus, as well as precisely regulate release profile during the combined effect of multiple stimuli [2-6].

Among various chemical and physical stimuli, phenylboronic acid (PBA) is a well known ligand that can form reversible covalent complexes with 1,2-cis-diol compounds, such as fructose in aqueous solution $[7,8]$. Based on this interaction, PBA functionalized polymers have been used for sugar sensing and drug delivery systems [9]. In particular, such polymers can potentially be developed into a self-regulating insulin delivery system to treat diabetes, where the more significant problem is the difficulty of controlling the insulin dosage. An insulin overdose can cause a low blood sugar level that may result in severe hypoglycemia $[10,11]$. To this end, a lot of sugar-sensitive linear copolymers [12, 13], latices [14], capsules [15-17], and hydrogels [18-21] were synthesized. For example, Kataoka et al. [8] synthesized a hydrogel composed of $\operatorname{poly}(N$ isopropylacrylamide) (PNIPAM) with PBA groups,

\footnotetext{
${ }^{*}$ Corresponding author, e-mail: tycang@mail.ahnu.edu.cn
} (c) BME-PT 
which shows an on-off regulation of insulin release in response to glucose stimulation at physiological $\mathrm{pH}$.

Controlled radical polymerization methods, such as atom-transfer radical polymerization (ATRP) and reversible addition-fragmentation chain transfer (RAFT) polymerization enabling the facile synthesis of a wide range of functional copolymers with controllable block lengths [22], have been used to synthesize boronic acid-containing block copolymers. Well-defined boronic acid homo- and block copolymers were synthesized via ATRP [23-25] or RAFT [26] polymerization of styrenic boronic ester and subsequent post-polymerization deprotection of the boronic ester. Recently, Sumerlin and coworkers $[3,27]$ reported the synthesis of well-defined PAAPBA-b-PNIPAM block copolymers via direct RAFT polymerization of unprotected AAPBA and NIPAM. Such copolymer is capable of forming micelles or reverse micelles depending on the temperature, $\mathrm{pH}$, and glucose concentration. However, these studies mainly focused on the characterization of their equilibrium self-assembled structures. Much less has been explored on the dissociation behavior of PAAPBA-b-PNIPAM micelles.

The study of the dissociation process of polymeric micelle is of fundamental importance for various applications, such as in dispersant technology and controlled drug release [28]. Polymeric micelles are expected to grow or dissolve primarily via Aniansson-Wall (AW) mechanism [29]. This mechanism, which allows change of the aggregation number only in steps consisting of addition or subtraction of individual chains into/out of the micelle, is found to have the lowest activation free energy. However, Zhang et al. [30] proposed that increasing the hydrophobic block length in block copolymers can transform the mechanism from unimer insertion/expulsion to micelle fusion/fission. Consequently, the micellization and dissociation of polymer has attracted considerable attention both in experimental and theoretical investigations [31-34].

In the present work, we prepared PAAPBA-b-PNIPAM block copolymer by direct RAFT polymerization. The micellization and dissociation transition of PAAPBA-b-PNIPAM in aqueous solution was systematically investigated by laser light scattering (LLS) in response to temperature, $\mathrm{pH}$, and fructose.
Our aim is to understand the dissociation behavior of micelles upon external stimuli.

\section{Experimental section \\ 2.1. Materials}

3-Aminophenylboronic acid was purchased from Alfa Aesar (Tianjin, China), and used as received. N-isopropylacrylamide (NIPAM, Aldrich, Shanghai, China) was recrystallized from a benzene $/ n-$ hexane mixture. Other chemicals were purchased from Shanghai Chemical Reagent Co. (Shanghai, China). Tetrahydrofuran (THF) was distilled from a purple sodium benzophenone ketyl solution. Acryloyl chloride, methanol and triethylamine were dried over $\mathrm{CaCl}_{2}$ and distilled. 4,4'-Azobis(isobutyronitrile) (AIBN) was purified by recrystallization from methanol. Phosphate-buffered saline (PBS, ionic strength $0.02 \mathrm{M}$ ) with various $\mathrm{pH}$ values was prepared by dissolving $\mathrm{KH}_{2} \mathrm{PO}_{4}, \mathrm{Na}_{2} \mathrm{HPO}_{4}$ and $\mathrm{Na}_{3} \mathrm{PO}_{4}$ in Milli-Q water with a resistivity of $18.3 \mathrm{M} \Omega \cdot \mathrm{cm}$. RAFT agent S-1-dodecyl-S'-( $\alpha, \alpha^{\prime}$-dimethyl- $\alpha^{\prime \prime}$-acetic acid) trithiocarbonate (CTA) was synthesized as described in the literature [35]. AAPBA, PAAPBA and PAAPBA-b-PNIPAM were synthesized according to the literature methods $[3,27]$. The polydispersity indices $\left(M_{\mathrm{w}} / M_{\mathrm{n}}\right)$ of PAAPBA and PAAPBA-bPNIPAM determined by Gel Permeation Chromatography (GPC) are 1.05 and 1.28, respectively.

\subsection{Preparation of the micellar solutions}

PAAPBA-b-PNIPAM $(2.0 \mathrm{mg})$ dissolved in $2 \mathrm{~mL}$ methanol was added to $100 \mathrm{~mL}$ water under stirring at a constant rate by an SP100i syringe pump. The methanol was removed by evaporation under reduced pressure. For the fructose sensitivity experiments, fructose was added to the polymer solutions. The $\mathrm{pH}$ of solution was adjusted to desired value by adding PBS stock solution with the final concentration of the block copolymer of $2.0 \times 10^{-5} \mathrm{~g} / \mathrm{mL}$. Each solution was incubated overnight at room temperature with continuous stirring and then was filtered through $0.45 \mu \mathrm{m}$ Millipore Millex-LCR filter to remove dust before the LLS measurement.

\subsection{Characterizations}

\subsubsection{LLS measurements}

All LLS measurements were conducted on an ALV/ DLS/SLS-5022F spectrometer with a multi- $\tau$ digi- 
tal time correlation (ALV5000, Germany) and a cylindrical $22 \mathrm{~mW}$ UNIPHASE He-Ne laser $\left(\lambda_{0}=\right.$ $632 \mathrm{~nm}$ ) as the light source. In static LLS [36], the weight-average molar mass $\left(M_{\mathrm{w}}\right)$ and the rootmean-square radius of gyration $\left\langle R_{\mathrm{g}}{ }^{2}>_{\mathrm{z}}{ }^{1 / 2}\right.$ (or written as $<R_{\mathrm{g}}>$ ) were obtained in a very solution from the angular dependence of the absolute excess timeaverage scattering intensity, known as the Rayleigh ratio $R_{\mathrm{vv}}(q)$ as shown by Equation (1):

$\frac{K C}{R_{\mathrm{vv}}(q)} \approx \frac{1}{M_{\mathrm{w}}}\left(1+\frac{1}{3}<R_{\mathrm{g}}^{2}>_{\mathrm{z}} q^{2}\right)$

where $K=4 \pi^{2} n^{2}(\mathrm{~d} n / \mathrm{d} C)^{2} /\left(N_{\mathrm{A}} \lambda_{0}^{4}\right)$ and $q=$ $4 \pi n \sin (\theta / 2) / \lambda_{0}$ with $C, \mathrm{~d} n / \mathrm{d} C, N_{\mathrm{A}}$, and $\lambda_{0}$ being concentration of the polymer, the specific refractive index increment, the Avogadro's number, and the wavelength of light, respectively. In dynamic LLS [37], the Laplace inversion of each measured intensity-intensity time correlation function $G^{(2)}(q, t)$ in the self-beating mode can lead to a line-width distribution $G(\Gamma)$. For a diffusive relaxation, $\Gamma$ is related to the translational diffusion coefficient $D$ by $\left(\Gamma / \mathrm{q}^{2}\right)_{\mathrm{C} \rightarrow 0, \mathrm{q} \rightarrow 0} \rightarrow D$, so that $G(\Gamma)$ can be converted into transitional diffusion coefficient distribution $G(D)$ and further into hydrodynamic radius distribution $f\left(R_{\mathrm{h}}\right)$ via the Stokes-Einstein equation, $R_{\mathrm{h}}=$ $k_{\mathrm{B}} T /(6 \pi \eta D)$, where $k_{\mathrm{B}}, T$, and $\eta$ are the Boltzmann constant, the absolute temperature, and the solvent viscosity, respectively. All DLS measurements were performed at a scattering angle $(\theta)$ of $20^{\circ}$. The refractive index increment $(\mathrm{d} n / \mathrm{d} C)$ value of micelle was measured by using a differential refractometer developed by Wu and Xia [38].

\subsubsection{GPC, NMR and TEM analyses}

The polydispersity indices of PAAPBA and PAAPBA-b-PNIPAM were determined by GPC (Waters 1515, Waters Corporation, USA) using monodisperse polystyrene as the calibration standard and THF as the eluent with a flow rate of $1.0 \mathrm{~mL} / \mathrm{min}$. The proton nuclear magnetic resonance $\left({ }^{1} \mathrm{H}-\mathrm{NMR}\right)$ spectra were recorded on a Bruker DMX-300 NMR spectrometer (Switzerland), using $\mathrm{CD}_{3} \mathrm{OD}$ as solvent and tetramethylsilane as an internal standard. The morphology of micelle was characterized by transmission electron microscopy (TEM). Samples were collected on a TEM grid and conducted using a Tecnai G2 20 S-TWIN (Holland) microscope with an accelerating voltage of $200 \mathrm{kV}$.

\section{Results and discussion}

\subsection{Synthesis and characterization of PAAPBA-b-PNIPAM copolymer}

Polymeric boronic acids are difficult to characterize by GPC because of extremely hygroscopic property [26]. Therefore, the compositions of PAAPBA and PAAPBA-b-PNIPAM are determined from ${ }^{1} \mathrm{H}$ NMR spectra. In the spectrum of PAAPBA-CTA (Figure 1a), the characteristic signals of both PAAPBA and CTA can be clearly observed. By calculating the intensity ratio of the signal at $\delta=6.9-7.8 \mathrm{ppm}$ to that at $\delta=1.16-1.26 \mathrm{ppm}$, which are assigned to phenyl protons in PAAPBA segment and methylene protons $\left(-\left(\mathrm{CH}_{2}\right)_{10}-\right)$ in CTA, respectively, the degree of polymerization (DP) of PAAPBA is evaluated to be 23 . From Figure 1b, the DP of PNIPAM block is determined to be 220 by comparing the intensity ratio of the signals of phenyl protons in PAAPBA and isopropyl protons in PNIPAM $(\delta=4.0 \mathrm{ppm})$.

\subsection{Thermo-responsive property of PAAPBA-b-PNIPAM micelles}

PNIPAM is one of the most popular thermo-responsive polymers, which undergoes a reversible phase transition at a lower critical solution temperature (LCST) around $32^{\circ} \mathrm{C}$ in water [39]. PNIPAM is soluble in water at temperature below its LCST, while PAAPBA is water insoluble in acidic or natural solution. Therefore, PAAPBA-b-PNIPAM chains can self-assemble to form a core-shell structure micelle with a hydrophobic core composed of PAAPBA and hydrophilic shell composed of PNIPAM in the lower $\mathrm{pH}$ solution at room temperature. To eliminate the effect of hydrophobic dodecyl group at the end of PAAPBA-b-PNIPAM chain on the self-assembly, the trithiocarbonate end-group of copolymer is cleaved by radical induced termination reaction with AIBN [40]. Figure 2 shows typical hydrodynamic radius distributions $f\left(R_{\mathrm{h}}\right)$ of PAAPBA-bPNIPAM. Only one unimodal distribution is observed at 20 and $37^{\circ} \mathrm{C}$, respectively. At $20^{\circ} \mathrm{C}$, an average hydrodynamic radius $\left(<R_{\mathrm{h}}>\right) 65 \mathrm{~nm}$ is calculated by CONTIN analysis; correspondingly, the average radius of gyration $\left.\left(<R_{\mathrm{g}}\right\rangle\right)$ of the scattering objects is $59 \mathrm{~nm}$ by static light scattering. It is known that the $<R_{\mathrm{g}}>/<R_{\mathrm{h}}>$ value can reveal the conformation of polymer chains or structure of particles. For random coil, hyper-branched cluster or micelle, and uniform sphere, $\left\langle R_{\mathrm{g}}>\right|<R_{\mathrm{h}}>$ is $1.5-1.8,1.0-1.2$, and 
b)
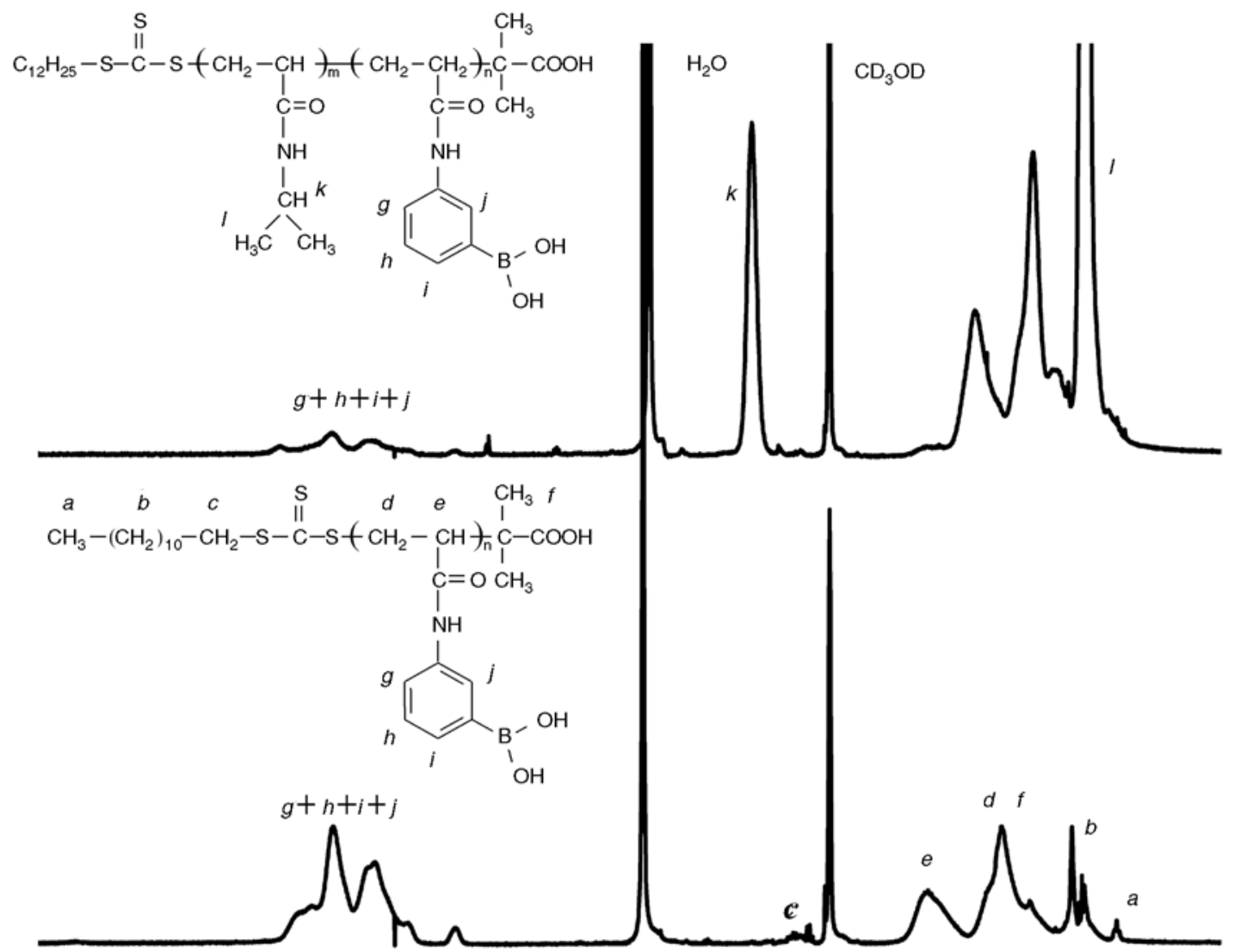

a)

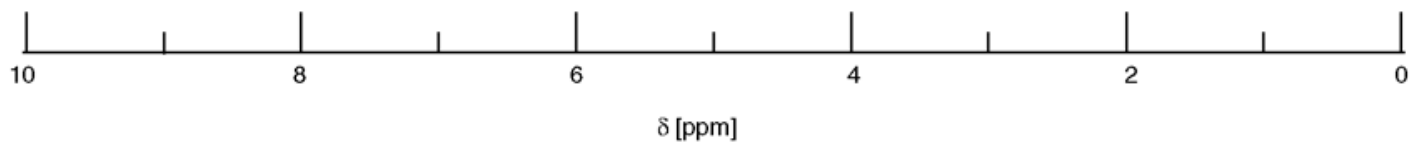

Figure 1. ${ }^{1} \mathrm{H}$ NMR spectra of (a) PAAPBA-CTA and (b) PAAPBA-b-PNIPAM in $\mathrm{CD}_{3} \mathrm{OD}$



Figure 2. Typical hydrodynamic radius distribution $\mathrm{f}\left(\mathrm{R}_{\mathrm{h}}\right)$ of PAAPBA-b-PNIPAM micelles ( $\mathrm{pH}$ 6.2) at 20 and $37^{\circ} \mathrm{C}$, respectively

$\sim 0.774$, respectively [41]. The ratio of $\left\langle R_{\mathrm{g}}>/<R_{\mathrm{h}}>\right.$ is 0.91 at $20.0^{\circ} \mathrm{C}$, indicating that PAAPBA-b-PNIPAM block copolymer forms micelles. However, the contour length of PAAPBA-b-PNIPAM chain is estimated to be $\sim 61 \mathrm{~nm}$ by $L=N l$, where $N$ is the total number of units, and the length per unit $l=0.25 \mathrm{~nm}$. Considering that the chains cannot be fully stretched in solution, their size should be much less than $61 \mathrm{~nm}$. We thus tentatively ascribe the formation of large irregular micelles. As the temperature increases to $37^{\circ} \mathrm{C}$, the $f\left(R_{\mathrm{h}}\right)$ becomes narrow with $<R_{\mathrm{h}}>28 \mathrm{~nm}$, suggesting the collapse of micelles. We note that besides the micelles in solution, there must be a small amount of free PAAPBA-b-PNIPAM chains, which are usually not detectable in the presence of micelles by DLS [42]. The actual morphology of the micelles has been obtained by TEM observation (Figure 3), which clearly reveals the presence of irregular micelles with average diameters of $50 \sim 65 \mathrm{~nm}$ at $20^{\circ} \mathrm{C}$ and spherical micelles with average diameters of $20 \sim 30 \mathrm{~nm}$ at $37^{\circ} \mathrm{C}$, respectively. The micelle sizes estimated from TEM were systematically smaller than those obtained by dynamic LLS, which is reasonable considering that the for- 


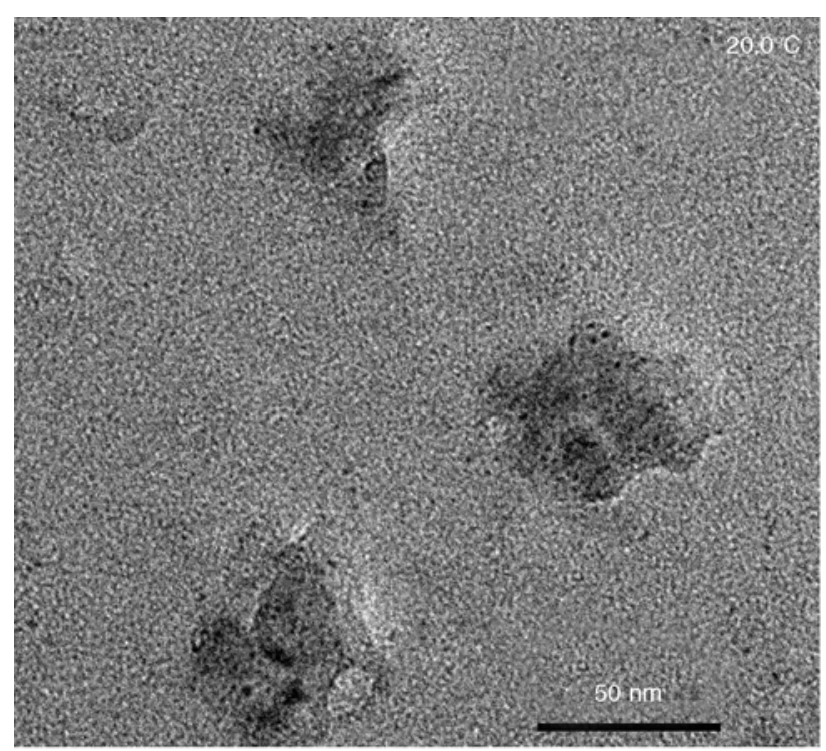

a)

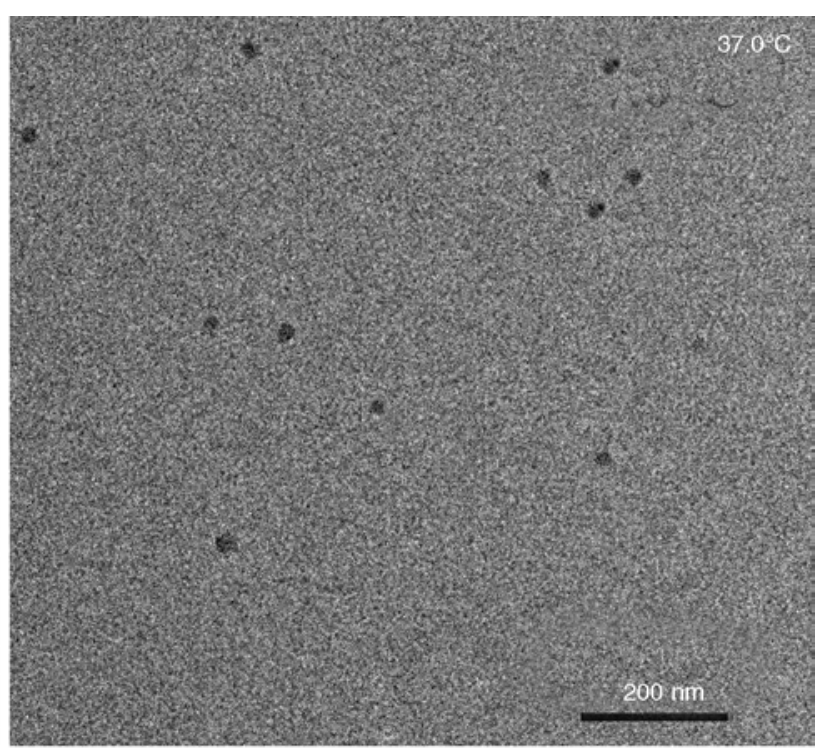

b)

Figure 3. TEM images of PAAPBA-b-PNIPAM micelles (pH 6.2) at (a) $20^{\circ} \mathrm{C}$ and (b) $37^{\circ} \mathrm{C}$, respectively

mer reflects conformations in the dry state, while the later reports the intensity-average dimensions of micelles in solution.

Figure 4 shows the temperature dependence of $<R_{\mathrm{h}}>$ and $<R_{\mathrm{g}}>$ of PAAPBA-b-PNIPAM micelles at $\mathrm{pH} 6.2$, where almost all of the boronic acid groups would be in the uncharged form [13]. Note that LLS measurements were carried out after the solution temperature reached equilibrium. At $T<30.0^{\circ} \mathrm{C}$, both $<R_{\mathrm{h}}>$ and $\left\langle R_{\mathrm{g}}>\right.$ slightly decrease with temperature, whereas the apparent molar mass $\left(M_{\mathrm{w}, \mathrm{a}}\right)$ remains nearly constant (inset of Figure 4), indicating micelles gradual shrink. In the range of $30.0-34.0^{\circ} \mathrm{C}$, the sharp decrease of $\left\langle R_{\mathrm{h}}>\right.$ and $\left\langle R_{\mathrm{g}}\right\rangle$ is the result of shrink and collapse of PNIPAM shell. On the contrary, $M_{\mathrm{w}, \mathrm{a}}$ increases from 1.05 to $1.52 \cdot 10^{7} \mathrm{~g} / \mathrm{mol}$. As we know

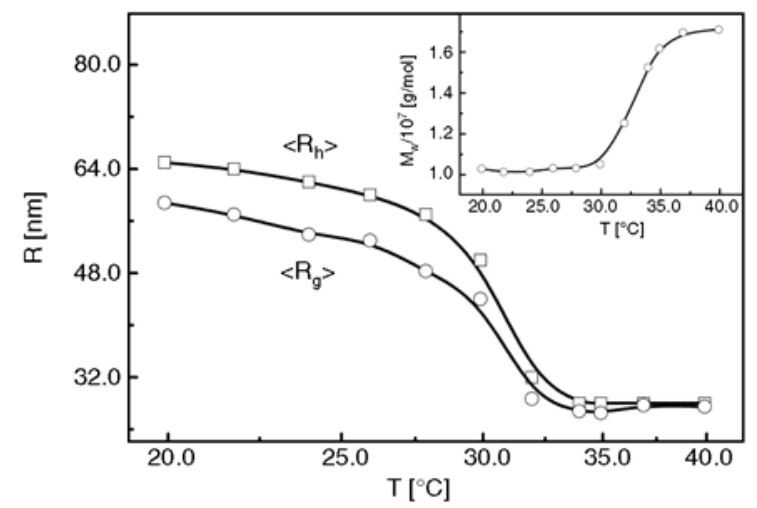

Figure 4. Temperature dependence of average hydrodynamic radius $\left(<R_{\mathrm{h}}>\right)$ and average radius of gyration $\left(<R_{\mathrm{g}}>\right)$ of PAAPBA-b-PNIPAM micelles at $\mathrm{pH}$ 6.2. The inset shows the temperature dependence of apparent molar mass $\left(M_{\mathrm{w}, \mathrm{a}}\right)$. that, the intrachain collapse and the interchain association compete with each other in the PNIPAM phase transition. The latter can cause significant change in $M_{\mathrm{w}, \mathrm{a}}$. For instance, if two micelles are associated, $M_{\mathrm{w}, \mathrm{a}}$ of new aggregates is nearly double that of micelles. Therefore, the small increase of $M_{\mathrm{w}, \mathrm{a}}$ might reflect that some free PAAPBA-b-PNIAPM chains in solution insert or small aggregates incorporate into the micelles. At the temperature above $34.0^{\circ} \mathrm{C},\left\langle R_{\mathrm{h}}>\right.$ and $<R_{\mathrm{g}}>$ slightly decrease to a constant value, and $M_{\mathrm{w}, \mathrm{a}}$ gradually levels off. The facts show that both the intrachain contraction of PNIPAM segments and individual chain inserted into the micelles stop. Note that no precipitation occurs even when the solution was heated up to $40.0^{\circ} \mathrm{C}$ for $20 \mathrm{~h}$, indicating that the micelles are stable.

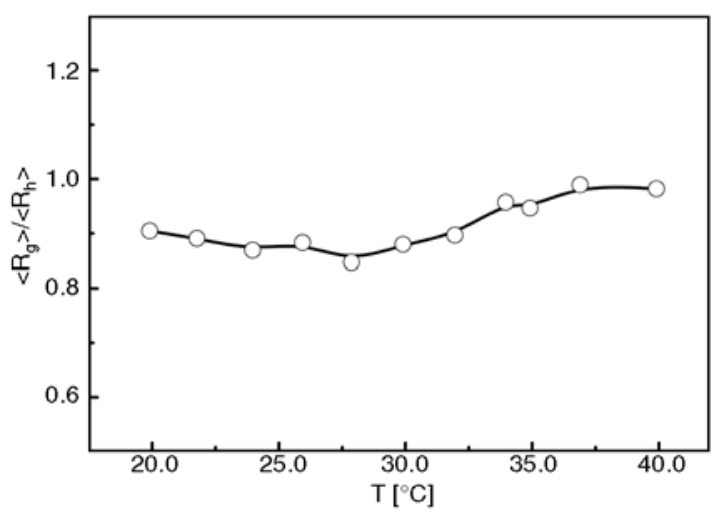

Figure 5. Temperature dependence of the ratio of average radius of gyration to average hydrodynamic radius $\left(<R_{\mathrm{g}}>/<R_{\mathrm{h}}>\right)$ of PAAPBA-b-PNIPAM micelles at $\mathrm{pH} 6.2$ 
Figure 5 shows that the ratio of $\left\langle R_{\mathrm{h}}>/<R_{\mathrm{g}}>\right.$ is $\sim 0.90$ 0.99 in the range of $20.0-40.0^{\circ} \mathrm{C}$, further indicating that PAAPBA-b-PNIPAM block copolymer forms micelles at all temperatures investigated. By meticulously analyzing the ratio change, we discover it slightly increases from 0.85 to 0.99 at $28.0-40.0^{\circ} \mathrm{C}$. The collapse of PNIPAM block causes the micellar shell density increase, which is responsible for the increase of $\left\langle R_{\mathrm{h}}\right\rangle /<R_{\mathrm{g}}>$ value.

\subsection{The dissociation behavior of PAAPBA-b-PNIPAM micelles upon $\mathbf{p H}$ and fructose stimulation}

There is an equilibrium between the different states of a phenylboronic acid derivative. As shown in Figure 6, with an increase in $\mathrm{pH}$ value, the neutral PBA can be ionized by combining with $\mathrm{OH}^{-}$to form a relatively hydrophilic but unstable phenylborate anion. Worth noticing is that the charged phenylborate can form a more favorable charged and stable phenylborate ester by complex with 1,2-cis-diol such as glucose in aqueous medium, and further addition of diol induces the shift of the equilibrium in the direction of increasing phenylborate ester [8, 9, 18]. Consequently, the water solubility of a polymer with PBA residues is tunable by changes in both $\mathrm{pH}$ and diol concentration.

Figure $7 \mathrm{a}$ shows $\mathrm{pH}$ dependence of $f\left(R_{\mathrm{h}}\right)$ of PAAPBA-b-PNIPAM micelles in the absence of<smiles>[R]c1cccc(B(O)O)c1</smiles><smiles>[R]c1cccc(B(O)O)c1</smiles>


Figure 6. Complexation equilibrium between PBA derivative and 1,2-diol

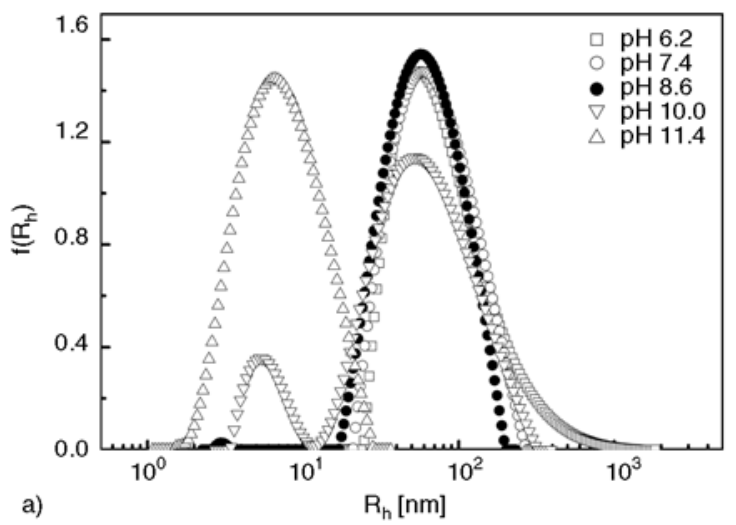

fructose at $20.0^{\circ} \mathrm{C}$. Compared with the micelles at $\mathrm{pH} 6.2$, the $f\left(R_{\mathrm{h}}\right)$ for the micelles at $\mathrm{pH} 7.4$ do not markedly change. Interestingly, a bimodal distribution can be observed at $\mathrm{pH} 8.6$ and 10.0, respectively. The big peak at $\sim 59 \mathrm{~nm}$ corresponding to the copolymer micelles is almost independent of $\mathrm{pH}$, suggesting that the micelle size remains unchanged. The new peak located at $\sim 3 \mathrm{~nm}$ is attributed to free chains of PAAPBA-b-PNIPAM. This bimodal distribution strongly suggests the coexistence of micelles and PAABA-b-PNIPAM free chains. As $\mathrm{pH}$ increases to 10.0 , the $f\left(R_{\mathrm{h}}\right)$ of the micelles decreases, while the scattering intensity of unimers increases. When the $\mathrm{pH}$ value is adjusted to 11.4 , we find only one peak located at $\sim 5 \mathrm{~nm}$, implying the complete dissociation of micelles. Since the $\mathrm{pKa}$ of AAPBA in PAAPBA-b-PNIPAM copolymer is around $8.2[9,10]$, phenylboronic acid group is partially charged, and completely charged at $\mathrm{pH} 8.6$ and 10.0, respectively [19]. As a result, more individual chains are dissociated from the copolymer micelles at higher $\mathrm{pH}$. Note that even if $\mathrm{pH}$ is increased up to10.0, which is much higher than the $\mathrm{p} K \mathrm{a}$ of PAAPBA, the micelles do not dissociate completely. Wang et al. [9] have reported that the micelles containing AAPBA block disaggregate at $\mathrm{pH}$ 11.3. This can be ascribed to the unique coreshell structure of micelles, where phenylborate moieties are spatially trapped in the micellar core. In the ionization process of PBA, the concentration of anionic charges in the immediate vicinity of phenylborates is increased and thus further dissociation of phenylborates is impeded. So much more $\mathrm{OH}^{-}$in the solution is needed to further ionize PBA groups in the inner core $[9,10]$.

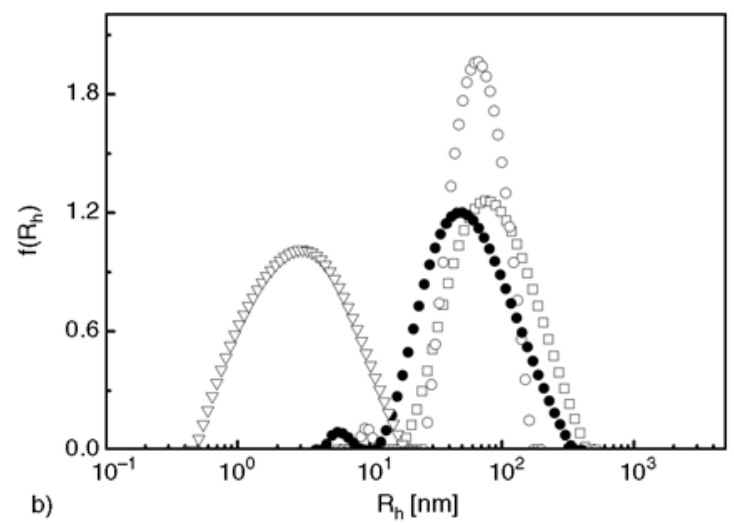

Figure 7. $\mathrm{pH}$ dependence of hydrodynamic radius distributions $\left(f\left(R_{\mathrm{h}}\right)\right)$ of PAAPBA-b- PNIPAM copolymer in (a) the absence and (b) the presence of fructose $\left(C_{\text {fructose }}=1.0 \mathrm{mg} / \mathrm{mL}\right)$ at $20^{\circ} \mathrm{C}$ 
The effect of fructose on the dissociation behavior of PAAPBA-b-PNIPAM micelles is studied by adding fructose $\left(C_{\text {fructose }}=1.0 \mathrm{mg} / \mathrm{mL}\right)$ to above copolymer solution with different $\mathrm{pH}$ values. As shown in Figure 7b, one similar unimodal distribution is observed at $\mathrm{pH} 6.2$ along with the peak shifting to the right direction, indicated that the micelles are swollen. At pH 7.4 and 8.6, some PAAPBA-bPNIPAM chains are dissociated from micelles, reflecting in a new peak; especially, at $\mathrm{pH} 10.0$, only one peak with $R_{\mathrm{h}} \sim 3 \mathrm{~nm}$ appears, suggesting that micelles are disappeared from the solution. As discussed above, the ionized PBA group number increases with $\mathrm{pH}$, and the charged PBA is thermodynamically favorable to bind with fructose to form phenylborate esters. As a result, the hydrophilicity of the micellar core increases with the addition of fructose, resulting in swelling and gradual disaggregation of the micelles.

Figure 8 shows the variation of $R_{\mathrm{h}}$ of PAAPBA-bPNIPAM micelles with or without fructose under different $\mathrm{pH}$, where $R_{\mathrm{h}}$ is corresponding to the peak shown in Figure 7. Clearly, increasing $\mathrm{pH}$ dramatically decreases $R_{\mathrm{h}}$ with the addition of fructose. On the other hand it causes $R_{\mathrm{h}}$ relatively small change in the absence of fructose. At pH 6.2 and 7.4, the presence of fructose increases the hydrophilicity of copolymer, and leads to the micelles swelling, reflecting in $R_{\mathrm{h}}$ increase, respectively. However, the $R_{\mathrm{h}}$ decreases at $\mathrm{pH}$ 8.4. Ge et al. [19] reported that the size of NIPAM-co-AAPBA microgel decreases with the addition of glucose. They attributed that glucose forms bidentate complexes with boronic acid groups and cross-links the segments between two chemical cross-linking sites,

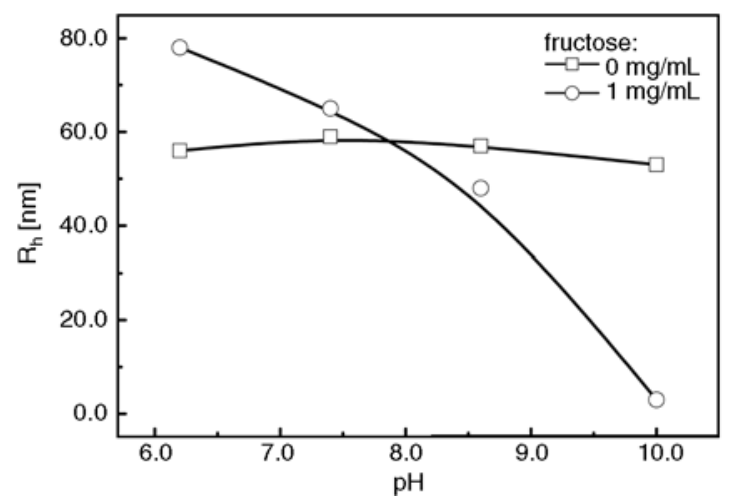

Figure 8. $\mathrm{pH}$ dependence of hydrodynamic radius $\left(R_{\mathrm{h}}\right)$ of PAAPBA-b-PNIPAM micelles (corresponding to the peaks shown in Figure 7) with and without fructose at $20.0^{\circ} \mathrm{C}$

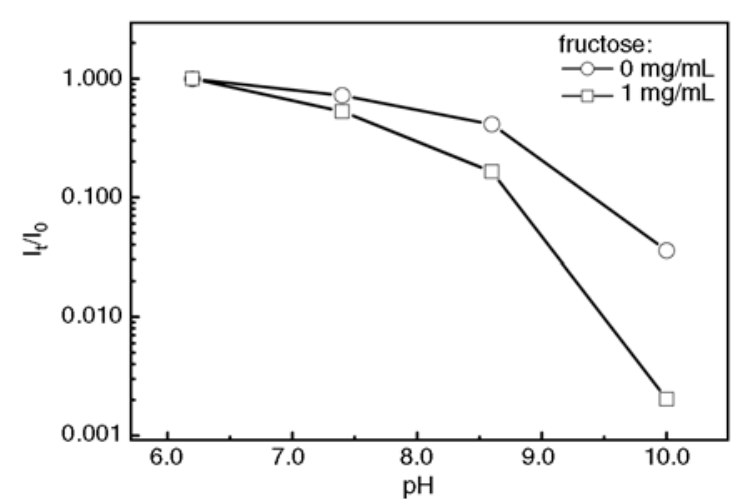

Figure 9. $\mathrm{pH}$ dependence of the relative scattering intensities $\left(I_{\mathrm{t}} / I_{0}\right)$ for PAAPBA-b-PNIPAM copolymer with and without fructose at $20.0^{\circ} \mathrm{C}$, where $I_{0}$ represents the scattering intensity at $\mathrm{pH} 6.2$, and $I_{\mathrm{t}}$ represents the scattering intensity at the specific $\mathrm{pH}$.

leading the microgels to shrink. It should be pointed out that fructose can only form mono (boronate) complexes with PBA group [18, 43]. Accordingly, the decrease in the micellar size is maybe because of the dissociation of unimer chains out of micelles. Figure 9 shows $\mathrm{pH}$ dependence of the relative scattering intensities $\left(I_{t} / I_{0}\right)$ for PAAPBA-b-PNIPAM micelles with and without fructose at $20.0^{\circ} \mathrm{C}$, where $I_{0}$ represents the scattering intensity of micelles at pH 6.2 in the absence of fructose, and $I_{\mathrm{t}}$ represents the scattering intensity at the specific $\mathrm{pH}$. It is well known that the scattering intensity is proportional to the square of the mass of a scattering object. The transition between micelles and individual chains can cause a significant change of $I_{\mathrm{t}}$. For the micelle solution without fructose, the ratio of $I_{\mathrm{t}} / I_{0}$ decreases as $\mathrm{pH}$ increase. In addition, this tendency becomes more significant when fructose is added. This further demonstrates that the addition of fructose is in favor of the formation of hydrophilic fructose phenylborate esters and promotes the swelling and then dissociation of the micelles. As fructose is added to $\mathrm{pH} 10.0$ solution, the effective solubility of PAAPBA segment leads to the complete dissociation of micelles.

In the present work, both the number and aggregation number (i.e. $M_{\mathrm{w}, \mathrm{a}}$ ) of micelles reduction can induce the scattering intensity to decrease. We think that the reduction of $M_{\mathrm{w}, \mathrm{a}}$ leads to both the ratio of $I_{\mathrm{t}} / I_{0}$ and the $R_{\mathrm{h}}$ of micelles decrease in the presence of fructose. As for the changes in $R_{\mathrm{h}}$ and the ratio of $I_{\mathrm{t}} / I_{0}$ with increasing $\mathrm{pH}$ in the absence of fructose, these are because of the simultaneous 
swelling and dissociation of micelles. Namely, the unimers are gradually expelled out of the micelles with the increase of $\mathrm{pH}$ and the addition of fructose. Furthermore, in the term of PAAPBA-b-PNIPAM structure, the DP of PAAPBA and PNIPAM block is 23 and 220, respectively. The diblock copolymers with longer shell-block lengths have a lower potential barrier for chain expulsion from micelles, which promotes more rapid chain exchange between micelles and solution [28, 30]. Accordingly, our results confirm that the micelle to unimer transformation process proceeds mainly via the unimer expulsion mechanism.

\section{Conclusions}

PAAPBA-b-PNIPAM block copolymers have been successfully synthesized by direct RAFT polymerization. Micelles self-assembled from PAAPBA-bPNIPAM exhibit temperature-, $\mathrm{pH}-$ and fructosesensitivity. Increasing the temperature to the LCST of PNIPAM leads to the micelles collapse. Adjustment of the solution $\mathrm{pH}$ from 6.2 to 10.0 at $20^{\circ} \mathrm{C}$ can result in the progressive dissociation of the micelles. Moreover, the addition of fructose increases the level of micelle dissociation. At $\mathrm{pH}$ 10.0 and the concentration of fructose $1 \mathrm{mg} / \mathrm{mL}$, the micelles completely dissociate to unimers. These results reveal that the main route of micelle dissociation involves step-by-step expulsion of unimers, namely, the dissociation of micelle via A-W mechanism. Owing to its property of the multiple micellziation and dissociation transitions, PAAPBA-b-PNIPAM holds great promise in designing stimuli-induced controlled release supports.

\section{Acknowledgements}

The financial support of the Natural Science Foundation of Anhui Province, China (11040606M61) and the Natural Science Foundation of the Anhui Higher Education Institutions of China (KJ2011A137, KJ2012A126) are gratefully acknowledged.

\section{References}

[1] Rapoport N.: Physical stimuli-responsive polymeric micelles for anti-cancer drug delivery. Progress in Polymer Science, 32, 962-990 (2007).

DOI: 10.1016/j.progpolymsci.2007.05.009
[2] Xia F., Ge H., Hou Y., Sun T., Chen L., Zhang G., Jiang L.: Multiresponsive surfaces change between superhydrophilicity and superhydrophobicity. Advanced Materials, 19, 2520-2524 (2007).

DOI: $10.1002 / \mathrm{adma} .200602334$

[3] Roy D., Cambre J. N., Sumerlin B. S.: Triply-responsive boronic acid block copolymers: Solution self-assembly induced by changes in temperature, $\mathrm{pH}$, or sugar concentration. Chemical Communication, 21062108 (2009).

DOI: $10.1039 / \mathrm{b} 900374 \mathrm{f}$

[4] Yu Y-Y., Tian F., Wei C., Wang C-C.: Facile synthesis of triple-stimuli (photo/pH/thermo) responsive copolymers of 2-diazo-1,2-naphthoquinone-mediated $\operatorname{poly}(\mathrm{N}$ isopropylacrylamide- $c o-N$-hydroxymethylacrylamide). Journal of Polymer Science Part A: Polymer Chemistry, 47, 2763-2773 (2009).

DOI: $10.1002 /$ pola. 23357

[5] Klaikherd A., Nagamani C., Thayumanavan S.: Multistimuli sensitive amphiphilic block copolymer assemblies. Journal of the American Chemical Society, 131, 4830-4838 (2009).

DOI: $10.1021 / \mathrm{ja} 809475 \mathrm{a}$

[6] Jin Q., Lv L-P., Liu G-Y., Xu J-P., Ji J.: Phenylboronic acid as a sugar- and $\mathrm{pH}$-responsive trigger to tune the multiple micellization of thermo-responsive block copolymer. Polymer, 51, 3068-3074 (2010).

DOI: $10.1016 /$ j.polymer.2010.04.061

[7] Kataoka K., Miyazaki H., Okano T., Sakurai Y.: Sensitive glucose-induced change of the lower critical solution temperature of poly[N,N-(dimethylacrylamide)co-3-(acrylamido)-phenylboronic acid] in physiological saline. Macromolecules, 27, 1061-1062 (1994). DOI: $10.1021 / \mathrm{ma} 00082 \mathrm{a} 028$

[8] Kataoka K., Miyazaki H., Bunya M., Okano T., Sakurai Y.: Totally synthetic polymer gels responding to external glucose concentration: Their preparation and application to on-off regulation of insulin release. Journal of the American Chemical Society, 120, 1269412695 (1998). DOI: $10.1021 /$ ja982975d

[9] Wang B., Ma R., Liu G., Liu X., Gao Y., Shen J., An Y., Shi L.: Effect of coordination on the glucose-responsiveness of PEG- $b$-(PAA-co-PAAPBA) micelles. Macromolecular Rapid Communications, 31, 16281634 (2010). DOI: $10.1002 /$ marc. 201000164

[10] Matsumoto A., Ikeda S., Harada A., Kataoka K.: Glucose-responsive polymer bearing a novel phenylborate derivative as a glucose-sensing moiety operating at physiological $\mathrm{pH}$ conditions. Biomacromolecules, 4, 1410-1416 (2003).

DOI: $10.1021 / \mathrm{bm} 034139 \mathrm{o}$

[11] Ravaine V., Ancla C., Catargi B.: Chemically controlled closed-loop insulin delivery. Journal of Controlled Release, 132, 2-11 (2008).

DOI: $10.1016 /$ j.jconrel.2008.08.009 
[12] Jin X., Zhang X., Wu Z., Teng D., Zhang X., Wang Y., Wang Z., Li C.: Amphiphilic random glycopolymer based on phenylboronic acid: Synthesis, characterization, and potential as glucose-sensitive matrix. Biomacromolecules, 10, 1337-1345 (2009).

DOI: $10.1021 / \mathrm{bm} 8010006$

[13] Shiomori K., Ivanov A. E., Galaev G. Y., Kawano Y., Mattiasson B.: Thermoresponsive properties of sugar sensitive copolymer of $\mathrm{N}$-isopropylacrylamide and 3(acrylamido)phenylboronic acid. Macromolecular Chemistry and Physics, 205, 27-34 (2004).

DOI: $10.1002 /$ macp. 200300019

[14] Cannizzo C., Amigoni-Gerbier S., Larpent C.: Boronic acid-functionalized nanoparticles: Synthesis by microemulsion polymerization and application as a re-usable optical nanosensor for carbohydrates. Polymer, 46, 1269-1276 (2005).

DOI: $10.1016 /$ j.polymer.2004.11.052

[15] Levy T., Déjugnat C., Sukhorukov G. B.: Polymer microcapsules with carbohydrate-sensitive properties. Advanced Functional Materials 18, 1586-1594 (2008). DOI: $10.1002 / \mathrm{adfm} .200701291$

[16] De Geest B. G., Jonas A. M., Demeester J., De Smedt S. C.: Glucose-responsive polyelectrolyte capsules. Langmuir, 22, 5070-5074 (2006).

DOI: $10.1021 / \mathrm{la} 0533680$

[17] Lapeyre V., Renaudie N., Dechezelles J-F., Saadaoui H., Ravaine S., Ravaine V.: Multiresponsive hybrid microgels and hollow capsules with a layered structure. Langmuir, 25, 4659-4667 (2009).

DOI: $10.1021 / 1 \mathrm{a} 9003438$

[18] Zhang Y., Guan Y., Zhou S.: Synthesis and volume phase transitions of glucose-sensitive microgels. Biomacromolecules, 7, 3196-3201 (2006).

DOI: $10.1021 / \mathrm{bm} 060557 \mathrm{~s}$

[19] Ge H., Ding Y., Ma C., Zhang G.: Temperature-controlled release of diols from $\mathrm{N}$-isopropylacrylamide$\mathrm{co}$-acrylamidophenylboronic acid microgels. The Journal of Physical Chemistry B, 110, 20635-20639 (2006). DOI: 10.1021/jp060914t

[20] Chen W., Pelton R., Leung V.: Solution properties of polyvinylamine derivatized with phenylboronic acid. Macromolecules, 42, 1300-1305 (2009).

DOI: $10.1021 / \mathrm{ma} 802402 \mathrm{z}$

[21] Liu P., Luo Q., Guan Y., Zhang Y.: Drug release kinetics from monolayer films of glucose-sensitive microgel. Polymer, 51, 2668-2675 (2010). DOI: $10.1016 /$ j.polymer.2010.04.011

[22] McCormick C. L., Lowe A. B.: Aqueous RAFT polymerization: Recent developments in synthesis of functional water-soluble (co)polymers with controlled structures. Accounts of Chemical Research, 37, 312-325 (2004).

DOI: $10.1021 / \operatorname{ar} 0302484$
[23] Qin Y., Cheng G., Sundararaman A., Jäkle F.: Well-defined boron-containing polymeric lewis acids. Journal of the American Chemical Society, 124, 12672-12673 (2002).

DOI: $10.1021 / \mathrm{ja} 020773 \mathrm{i}$

[24] Qin Y., Sukul V., Pagakos D., Cui C., Jäkle F.: Preparation of organoboron block copolymers via ATRP of silicon and boron-functionalized monomers. Macromolecules, 38, 8987-8990 (2005).

DOI: $10.1021 / \mathrm{ma} 051615 \mathrm{p}$

[25] Kim K. T., Cornelissen J. J. L. M., Nolte R. J. M., van Hest J. C. M.: A polymersome nanoreactor with controllable permeability induced by stimuli-responsive block copolymers. Advanced Materials, 21, $2787-$ 2791 (2009).

DOI: $10.1002 / \mathrm{adma} .200900300$

[26] Cambre J. N., Roy D., Gondi S. R., Sumerlin B. S.: Facile strategy to well-defined water-soluble boronic acid (co)polymers. Journal of the American Chemical Society, 129, 10348-10349 (2007).

DOI: $10.1021 / \mathrm{ja} 074239 \mathrm{~s}$

[27] Roy D., Cambre J. N., Sumerlin B. S.: Sugar-responsive block copolymers by direct RAFT polymerization of unprotected boronic acid monomers. Chemical Communications, 23, 2477-2479 (2008).

DOI: $10.1039 / \mathrm{b} 802293 \mathrm{c}$

[28] Li Z., Dormidontova E. E.: Kinetics of diblock copolymer micellization by dissipative particle dynamics. Macromolecules, 43, 3521-3531 (2010). DOI: $10.1021 / \mathrm{ma9} 92860 \mathrm{j}$

[29] Aniansson E. A. G., Wall S. N.: Kinetics of step-wise micelle association. Correction and improvement. Journal of Physical Chemistry, 79, 857-858 (1975). DOI: $10.1021 / \mathrm{j} 100575 \mathrm{a} 019$

[30] Zhang J., Xu J., Liu S.: Chain-length dependence of diblock copolymer micellization kinetics studied by stopped-flow pH-jump. Journal of Physical Chemistry Part B, 112, 11284-11291 (2008).

DOI: $10.1021 / \mathrm{jp} 803700 \mathrm{n}$

[31] Halperin A., Alexander S.: Polymeric micelles: Their relaxation kinetics. Macromolecules, 22, 2403-2412 (1989).

DOI: $10.1021 / \mathrm{ma} 00195 \mathrm{a} 069$

[32] Dormidontova E. E.: Micellization kinetics in block copolymer solutions: Scaling model. Macromolecules, 32, 7630-7644 (1999). DOI: $10.1021 / \mathrm{ma} 9809029$

[33] Nyrkova I. A., Semenov A. N.: On the theory of micellization kinetics. Macromolecular Theory and Simulations, 14, 569-585 (2005).

DOI: $10.1002 /$ mats.200500010

[34] Zhu Z., Xu J., Zhou Y., Jiang X., Armes S. P., Liu S.: Effect of salt on the micellization kinetics of $\mathrm{pH}$-responsive abc triblock copolymers. Macromolecules, 40, 6393-6400 (2007). DOI: $10.1021 / \mathrm{ma} 070978 \mathrm{~h}$ 
[35] Lai J. T., Filla D., Shea R.: Functional polymers from novel carboxyl-terminated trithiocarbonates as highly efficient RAFT agents. Macromolecules, 35, 67546756 (2002).

DOI: $10.1021 / \mathrm{ma} 020362 \mathrm{~m}$

[36] Berne B. J., Pecora R.: Dynamic light scattering. Wiley, New York (1976).

[37] Chu B., Laser light scattering. Academic Press, New York (1991).

[38] Wu C., Xia K-Q.: Incorporation of a differential refractometer into a laser light-scattering spectrometer. Review of Scientific Instruments, 65, 587-590 (1994). DOI: $10.1063 / 1.1145121$

[39] Winnik F. M.: Fluorescence studies of aqueous solutions of poly(N-isopropylacrylamide) below and above their LCST. Macromolecules, 23, 233-242 (1990).

DOI: $10.1021 / \mathrm{ma} 00203 \mathrm{a} 040$

[40] Perrier S., Takolpuckdee P., Mars C. A.: Reversible addition-fragmentation chain transfer polymerization: End group modification for functionalized polymers and chain transfer agent recovery. Macromolecules, 38, 2033-2036 (2005).

DOI: $10.1021 / \mathrm{ma} 047611 \mathrm{~m}$
[41] Douglas J. F., Roovers J., Freed K. F.: Characterization of branching architecture through 'universal' ratios of polymer solution properties. Macromolecules, 23, 41684180 (1990).

DOI: $10.1021 / \mathrm{ma} 00220 \mathrm{a} 022$

[42] Troll K., Kulkarni A., Wang W., Darko C., Koumba A. M. B., Laschewsky A., Müller-Buschbaum P., Papadakis C. M.: The collapse transition of poly(styrene- $b-(N-$ isopropyl acrylamide)) diblock copolymers in aqueous solution and in thin films. Colloid and Polymer Science, 286, 1079-1092 (2008).

DOI: $10.1007 / \mathrm{s} 00396-008-1871-5$

[43] Alexeev V. L., Sharma A. C., Goponenko A. V., Das S., Lednev I. K., Wilcox C. S., Finegold D. N., Asher S. A.: High ionic strength glucose-sensing photonic crystal. Analytical Chemistry, 75, 2316-2323 (2003). DOI: $10.1021 / \mathrm{ac} 030021 \mathrm{~m}$ 